\title{
10. RADIOLARIANS FROM THE MOROCCAN BASIN, DEEP SEA DRILLING PROJECT LEG 50
}

\author{
M. Jean Westberg, Annika Sanfilippo, and W. R. Riedel, Scrips Institution of Oceanography, \\ La Jolla, California
}

\begin{abstract}
At Sites 415 and 416, a few Miocene samples yielded identifiable radiolarians, permitting their placement in the established zonation. Two Paleocene cores from Site 415 contained radiolarians from a part of the section not yet zoned, and a few identifiable radiolarians were found in the Cretaceous Cores 415A-11 through 415A-13.
\end{abstract}

\section{INTRODUCTION}

Radiolarians occurred in parts of the sections at both sites drilled during DSDP Leg 50: Site 415 at $31^{\circ} 01.72^{\prime} \mathrm{N}$, $11^{\circ} 39.11^{\prime} \mathrm{W}$, water depth 2794 meters, and Site 416 at $32^{\circ} 50.18^{\prime} \mathrm{N}, 10^{\circ} 48.06^{\prime} \mathrm{W}$, water depth 4191 meters.

Rather poor radiolarian assemblages were found in the Cretaceous and Paleocene of Site 415. Miocene sequences of well-preserved radiolarian assemblages were sampled at both Sites 415 and 416 ; species occurrences are indicated in Tables 1 and 2.

\section{OCCURRENCES OF RADIOLARIANS}

The following paragraphs briefly describe the radiolarian occurrences. Figure 1 summarizes this information, in relation to the occurrences of calcareous microfossils, for the nearby Site 370 in addition to Sites 415 and 416. Apparent differences in radiolarian occurrences, between Site 370 and the adjacent Site 416, are probably not very significant. In the early and middle Eocene, radiolarians are rare and poorly preserved at Site 370 (David R. Johnson, personal communication) and absent at Site 416.

\section{Site $\mathbf{4 1 5}$}

Two samples from the Quaternary of Core 415-1 contained only very rare, poorly preserved orosphaerid fragments and indeterminate spumellarians.

Sample 415B-2-1, 60-62 cm yielded no radiolarians, but samples from the Pliocene and Miocene of Cores 415-2 through 415-5 contained a sufficient number of moderately preserved radiolarians for stratigraphic interpretation. The relative abundances of some species are shown in Table 1. The assemblage in Core 415-2 apparently belongs in the Stichocorys peregrina Zone of Riedel and Sanfilippo (1970, in press) or the Spongaster pentas Zone of Riedel and Sanfilippo (1970, in press), but the species necessary to distinguish between the two are not present. Cores 415-3 and 415-4 evidently belong in the Dorcadospyris alata Zone of Riedel and Sanfilippo $(1970,1971)$. Assemblages representing the Calocycletta costata Zone of Riedel and Sanfilippo (1970) probably occur in the 60 -meter unsampled gap between
Cores 415-4 and 415-5, and the assemblages of Core 415-5 belong in the Stichocorys wolffii Zone in Riedel and Sanfilippo (in press; the upper part of the former Calocycletta virginis Zone).

The early Eocene of Core 415A-1 contains no radiolarians, nor does the Paleocene of Core 415-A-4. Rare, poorly to moderately well preserved radiolarians occur in Cores 415A-5 and 415A-6, indicating the unzoned part of the Paleocene older than the Bekoma bidartensis Zone of Foreman (1973a): Amphiternis sp. cf. Stichomitra (?) alamedaensis (very rare to rare); Bekoma spp. fragments (very rare to few); $B$. campechensis (very rare); $B$. divaricata (very rare to rare); Buryella pentadica (rare to few); $B$. tetradica (few to common); Clathrocycloma spp. (rare); Lamptonium colymbus one specimen in 415A-5, CC); L. pennatum (rare); Lithomitra lineata (very rare); one or more species of Lychnocanoma with small thorax and abdomen joined to slightly divergent, bladed feet (Plate 1, Figures 11 and 12; rare); Phormocyrtis striata exquisita (rare to few); Pterocodon sp. (Plate 1, Figure 14; one specimen in 415A-6-2), with less inflated thorax than $P$. lex Sanfilippo and Riedel (in press); saturnalins with spiny rings (Plate 1, Figure 2; very rare to rare); Spongopyle insolita (one specimen in 415A-6-2); a spyrid with robustcylindrical feet (Plate 1, Figure 4; rare); Stylosphaera goruna (rare to few); and Stylotrochus alveatus (very rare to few).

Radiolarians are sparse and poorly preserved in the Cretaceous. In three samples from Core 415A-7 and the top of Core $415 \mathrm{~A}-8$, they are rare to very rare, filled with zeolite or redeposited silica, and not identifiable. In the lower part of Core 415A-8 through the top of Core $415 \mathrm{~A}-11$, nine samples yielded no radiolarians. From 415A-11-2 downward, radiolarians are very rare to rare and calcitized, and some forms are recognizable. The core-catcher sample of Core 415A-11 yielded a pseudoaulophacid Dictyomitra somphedia. Together, the four samples-415A-12-2, 53-55 cm; 415A-12, CC; 415A-13-1, 135-137 cm; and 415A-13-2, 64-66 cmyielded Amphipyndax stocki, Dictyomitra macrocephala, D. torquata, D. veneta, Eucyrtis bulbosus, E. tenuis, and Stichomitra asymbatos. Four samples from $415 \mathrm{~A}-13$, CC through $415 \mathrm{~A}-15$, CC contained very rare radiolarian fragments. 
TABLE 1

Abundances of Some Radiolarians from Hole 415

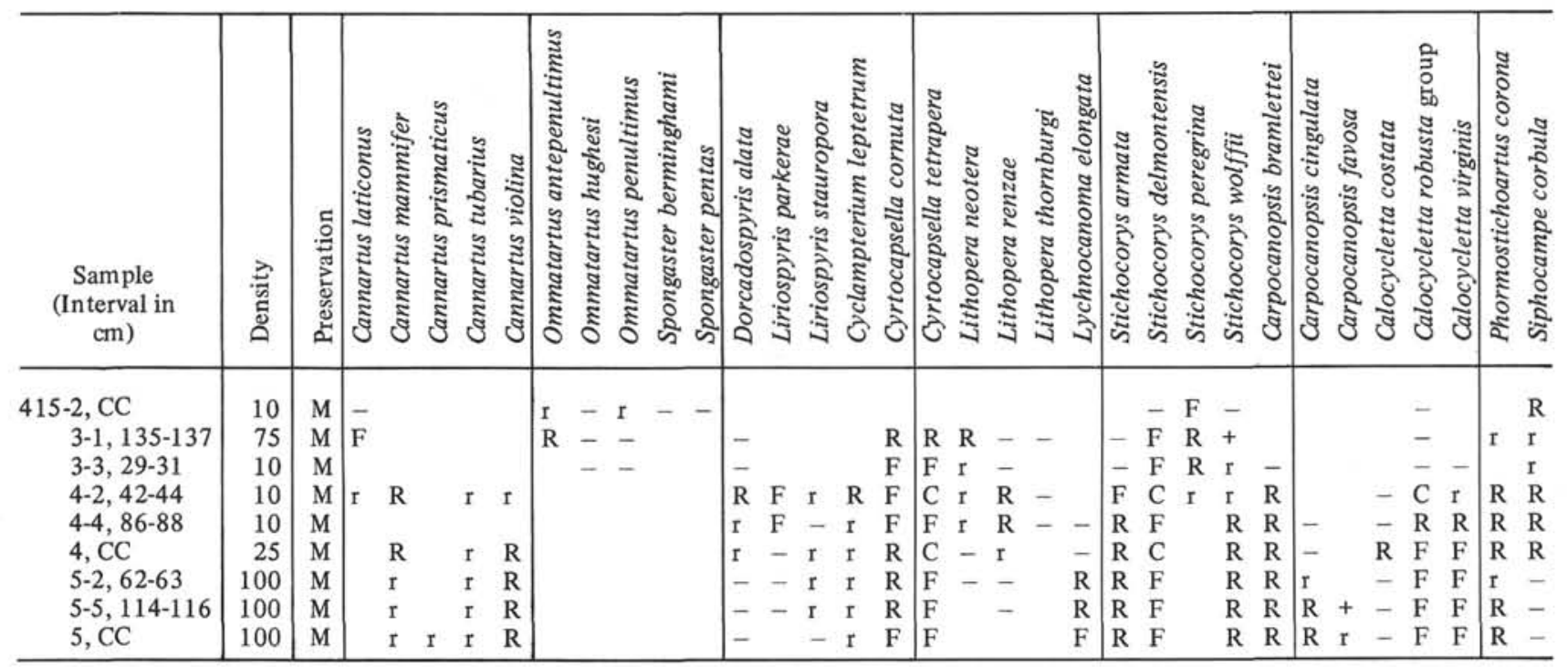

Note: In the Density/Preservation column: $M$ indicates moderate; $10,25,50,75$, and 100 reflect the average number of radiolarians per $0.35 \mathrm{~mm}^{2}$ field on the slide, as applied by Westberg and Riedel (in press). In the body of the table: - indicates species sought but not found; + indicates trace presence (estimated $<0.01 \%$ of the radiolarians on the slide); $\mathrm{r}$, very rare $(0.01$ to $0.1 \%) ; \mathrm{R}$, rare $(0.1$ to $1 \%)$; F, few (1 to $10 \%)$; and C, common $(>10 \%)$.

TABLE 2

Abundances of Some Radiolarians from Hole 416A

\begin{tabular}{|c|c|c|c|c|c|c|c|c|c|c|c|c|c|c|c|c|c|c|c|c|c|c|}
\hline $\begin{array}{c}\text { Sample } \\
\text { (Interval in } \mathrm{cm} \text { ) }\end{array}$ & $\begin{array}{l}\text { 焉 } \\
\text { ص }\end{array}$ & 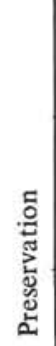 & 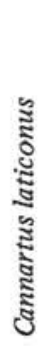 & 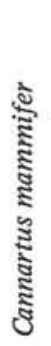 & 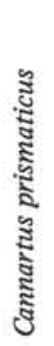 & 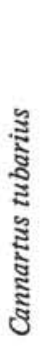 & 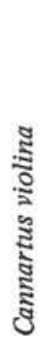 & 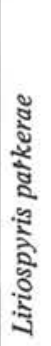 & 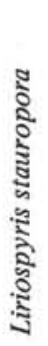 & 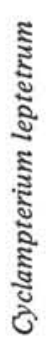 & 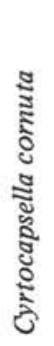 & 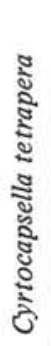 & 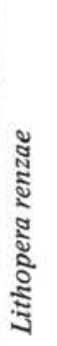 & 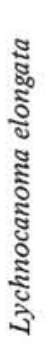 & 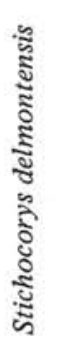 & 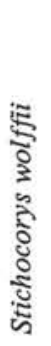 & 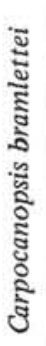 & 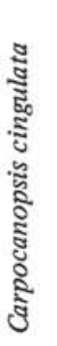 & 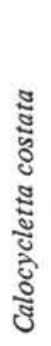 & 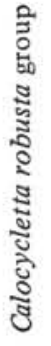 & 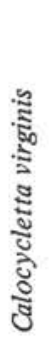 & 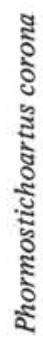 \\
\hline $16 \mathrm{~A}-1, \mathrm{C}$ & $<10$ & M & - & & & & & & & & $\mathrm{F}$ & $\mathrm{F}$ & & & $\mathrm{F}$ & & & & & & F & \\
\hline 2-1 & 10 & M & - & $\mathrm{R}$ & & & $\mathrm{R}$ & - & $\mathrm{R}$ & $\mathrm{R}$ & R & F & $\mathrm{R}$ & - & $\mathrm{F}$ & r & $\mathrm{r}$ & $\mathrm{R}$ & $\mathrm{r}$ & $\mathrm{R}$ & $\mathrm{R}$ & r \\
\hline $2-1,125-$ & 10 & M & & $\mathrm{R}$ & & & $\mathrm{F}$ & & - & & $\mathrm{R}$ & $\mathrm{F}$ & $R$ & - & & & & $\mathrm{R}$ & - & & $\mathrm{R}$ & - \\
\hline $2-1,126-127$ & 10 & M & & $\mathrm{R}$ & & & $\mathrm{R}$ & - & I & & $\mathrm{R}$ & $\mathrm{F}$ & $\mathrm{R}$ & - & $\mathrm{F}$ & $\mathrm{R}$ & $\mathrm{R}$ & $\mathrm{R}$ & $\mathrm{r}$ & $\mathrm{F}$ & $\mathrm{F}$ & - \\
\hline $2-3,40-42$ & 10 & M & & $\mathrm{R}$ & & & $\mathrm{R}$ & & r & $\mathrm{R}$ & $\mathrm{R}$ & $\mathrm{F}$ & $\mathrm{R}$ & r & $\mathrm{F}$ & $\mathrm{R}$ & $\mathrm{R}$ & $\mathrm{R}$ & - & $\mathrm{R}$ & $\mathrm{R}$ & $\mathrm{r}$ \\
\hline $2, \mathrm{CC}$ & 10 & M & & $\mathrm{R}$ & $\mathrm{R}$ & $\mathrm{R}$ & F & & & & $\mathrm{R}$ & $\mathrm{F}$ & $\mathrm{R}$ & $\mathrm{R}$ & $\mathrm{F}$ & $\mathrm{R}$ & $\mathrm{R}$ & & $\mathrm{R}$ & $\mathrm{R}$ & $\mathrm{R}$ & - \\
\hline
\end{tabular}

Note: Symbols as in Table 1 .

\section{Site 416}

Four samples from Cores 416-1 and 416-2 yielded very rare, indeterminable radiolarian fragments.

Core 416A-1 contained rare, rather long-ranging radiolarians, and Core 416A-2 contained radiolarians from near the boundary between the Calocycletta costata Zone and the Stichocorys wolfii Zone (see Table 2). Core 416A-2 also contained a little reworked late Eocene and Oligocene, indicated by rare specimens of Artophormis gracilis, Cyclampterium pegetrum, Lithocyclia aristotelis group, Theocorys spongoconum, and Theocyrtis annosa. The turbidites in Core 416A-3 contain very rare, generally corroded radiolarians of mixed age. Core 416A-3 turbidites include Stichocorys del- montenesis, Dorcadospyris ateuchus, Cyclampterium pegetrum, Theocampe mongolfieri, Amphisphaera minor, Lithomitra docilis, and Dictyomitra spp.

Ninety-seven samples examined from Cores 416A-4 through 416A-57 yielded no radiolarians.

\section{SPECIES LIST}

Amphipyndax stocki (Campbell and Clark) Stichocapsa (?) stocki Campbell and Clark, 1944b, p. 44, pl. 8, fig. 31-33.

Amphipyndax stocki (Campbell and Clark), Foreman, 1968, p. 78, pl. 8, fig. 12a-c.

Amphisphaera minor (Clark and Campbell)

Stylosphaera minor Clark and Campbell, 1942, p. 27, pl. 5, fig. 1, $2,2 \mathrm{a}, 12$. 


\begin{tabular}{|c|c|c|c|}
\hline Ages & $\begin{array}{l}\text { Site } \\
415\end{array}$ & $\begin{array}{c}\text { Site } \\
416\end{array}$ & $\begin{array}{l}\text { Site } \\
370\end{array}$ \\
\hline Quaternary & $\square$ & & 1 \\
\hline Pliocene & $\square$ B1, & & \\
\hline $\begin{array}{l}\text { Late } \\
\text { Miocene }\end{array}$ & & & 2 \\
\hline $\begin{array}{l}\text { Middle } \\
\text { Miocene }\end{array}$ & 3 & 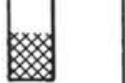 & 3 \\
\hline $\begin{array}{l}\text { Early } \\
\text { Miocene }\end{array}$ & 5 & $\mathrm{~A} 2$ & 4 \\
\hline Oligocene & & $A 3$ & 5 \\
\hline $\begin{array}{l}\text { Late } \\
\text { Eocene }\end{array}$ & & & 6 \\
\hline $\begin{array}{l}\text { Middle } \\
\text { Eocene }\end{array}$ & & A4 & 7-12 \\
\hline $\begin{array}{l}\text { Early } \\
\text { Eocene }\end{array}$ & A1 & A5 & $13-$ \\
\hline Paleocene & $\begin{array}{l}\mathrm{A} 4- \\
\mathrm{A} 6\end{array}$ & & \begin{tabular}{|l}
$\square$ \\
\end{tabular} \\
\hline Cretaceous & A7- & $\square_{\text {A57 }}^{A 5-}$ & $\square_{50}^{20}$ \\
\hline
\end{tabular}

Figure 1. Sediments cored on DSDP Leg 50, in the Moroccan Basin. Cores are represented by numbered rectangles. Empty rectangles represent cores containing calcareous fossils but lacking siliceous fossils, and hachuring indicates radiolarians occurring with calcareous fossils. The positions of the rectangles indicating cores do not always reflect uncertainties in age assignments; for details, see the contributions on the various fossil groups (this volume). Cores are indicated as containing radiolarians only if more than rare fragments are present.

Amphisphaera minor (Clark and Campbell), Sanfilippo and Riedel, 1973, p. 486, pl. 1, fig. 1-5; pl. 22, fig. 4.

Amphiternis sp. cf. (?) Stichomitra alamedaensis (Campbell and Clark)

cf. Phormocampe (Crytocorys) alamedaensis Campbell and Clark, 1944b, p. 37 , pl. 7, fig. 41 .

cf. (?) Stichomitra alamedaensis (Campbell and Clark), Foreman, 1968 , p. 77 , pl. 8 , fig. 4.

Artophormis gracilis Riedel

Artophormis gracilis Riedel, 1959, p. 300, pl. 2, fig. 12, 13.
Bekoma campechensis Foreman

Bekoma campechensis Foreman, 1973a, p. 432, pl. 3, fig. 24; pl. 10 , fig. 1, 2, 4.

Bekoma divaricata Foreman Bekoma divaricata Foreman, 1973a, p. 433, pl. 3, fig. 23; pl. 10, fig. 3, 4 .

Buryella pentadica Foreman

Buryella pentadica Foreman, 1973a, p. 433, pl. 8, fig. 8; pl. 9, fig. $15,16$.

Buryella tetradica Foreman

Buryella tetradica Foreman, 1973a, p. 433, pl. 8, fig. 4, 5; pl. 9, fig. 13,14 .

Calcoycletta costata (Riedel) Calocyclas costata Riedel, 1959, p. 296, pl. 2, fig. 9.

Calocycletta costata (Riedel), Riedel and Sanfilippo, 1970, p. 535, pl. 14 , fig. 12.

Calocycletta robusta Moore group Calocycletta robusta Moore, 1971, p. 743, pl. 10, fig. 5, 6. Calocycletta caepa Moore, 1972, p. 150, pl. 2, fig. 4-7.

Calocycletta virginis Haeckel Calocyclas virginis Haeckel, 1887, p. 1381, pl. 74, fig. 4. Calocycletta virginis Haeckel, Riedel, 1959, p. 295, pl. 2, fig. 8. Calocycletta virginis Haeckel, Riedel and Sanfilippo, in press.

Cannartus laticonus Riedel Cannartus laticonus Riedel, 1959, p. 291, pl. 1, fig. 5. Cannartus laticonus Riedel, Westberg and Riedel, in press.

Cannartus mammifer (Haeckel)

Cannartidium mammiferum Haeckel, 1887, p. 375, pl. 39, fig. 16.

Cannartus mammiferus (Haeckel), Riedel, 1959, p. 291, pl. 1, fig. 4.

Cannartus prismaticus (Haeckel) Pipettella prismatica Haeckel, 1887, p. 305, pl. 39, fig. 6 . Cannartus prismaticus (Haeckel). Riedel and Sanfilippo, 1970, p. 520 , pl. 15, fig. 1.

Cannartus tubariuis (Haeckel)

Pipettaria tubaria Haeckel, 1887, p. 339, pl. 39, fig. 15 Cannartus tubarius (Haeckel), Riedel, 1959, p. 289, pl. 1, fig. 2.

Cannartus violina (Haeckel) Cannartus violina Haeckel, 1887 , p. 358 , pl. 39 , fig. 10 . Cannartus violina Haeckel, Riedel, 1959, p. 290, pl. 1, fig. 3.

Carpocanopis bramlettei Riedel and Sanfilippo Carpocanopis bramlettei Riedel and Sanfilippo, 1971, p. 1597, pl. $2 \mathrm{G}$, fig. $8-14$; pl. 8 , fig. 7 .

Carpocanopsis cingulata Riedel and Sanfilippo Carpocanopsis cingulatum Riedel and Sanfilippo, 1971, p. 1597, pl. 2G, fig. $17-21$; pl. 8 , fig. 8 . Carpocanopsis cingulata Riedel and Sanfilippo, in press.

Carpocanopsis favosa (Haeckel)

Cycladophora favosa Haeckel, 1887, p. 1380, pl. 62, fig. 5, 6. Carpocanopsis favosum (Haeckel), Riedel and Sanfilippo, 1971, p. 1597, pl. 2G, fig. 15, 16; pl. 8, fig. 9-11.

Dyclampterium leptetrum Sanfilippo and Riedel Cyclampterium ? leptetrum Sanfilippo and Riedel, 1970, p. 456, p. 2, fig. 11,12 .

Cyclampterium pegetrum Sanfilippo and Riedel Cyclampterium? pegetrum Sanfilippo and Riedel, 1970, p. 456, p. 2 , fig. 8-10.

Cyclampterium pegetrum Sanfilippo and Riedel, Riedel and Sanfilippo, in press.

Cyrtocapsella cornuta Haeckel

Cyrtocapsa (Crytocapsella) cornuta Haeckel, 1887, p. 1513, p. 78, fig. 9.

Cytrocapsella cornuta Haeckel, Sanfilippo and Riedel, 1970, p. 453 , pl. 1, fig. 19, 20.

Cyrtocapsella tetrapera Haeckel Cyrtocapsa (Crytocapsella) tetrapera Haeckel, 1887, p. 1512, pl. 78, fig. 5 . Cyrtocapsella tetrapera Haeckel, Sanfilippo and Riedel, 1970, p. 453, pl. 1, fig. 16-18.

Dictyomitra macrocephala Squinabol Dicomitra macrocephala Squinabol, 1904, p. 230, pl. 9, fig. 10.

Dictyomitra somphedia Foreman Dictyomitra somphedia Foreman, 1973b, p. 264, pl. 14, fig. 18.

Dictyomitra torquata Foreman Dictyomitra torquata Foreman, 1971, p. 1676, p. 3, fig. 4. 
Dictyomitra torquata Foreman, Riedel and Sanfilippo, 1974, p. 778 , pl. 5, fig. 1-4; pl. 14, fig. 2.

Dictyomitra veneta (Squinabol)

Phormocyrtis veneta Squinabol, 1903, p. 134, pl. 9, fig. 30.

Dictyomitra veneta (Squinabol), Petrushevskaya and Kozlova, 1972, p. 550, pl. 2, fig. 2.

Dictyomitra veneta (Squinabol), Riedel and Sanfilipp, 1974, p. 778 , pl. 5, fig. 5, 6 .

Dorcadospyris alata (Riedel)

Brachiospyris alata Riedel, 1959, p. 293, pl. 1, fig. 11, 12. Dorcadospyris alata (Riedel), Riedel and Sanfilippo, 1970, p. 523, p. 14 , fig. 5 .

Dorcadospyris ateuchus (Ehrenberg)

Ceratospyris ateuchus Ehrenberg, 1873, p. 218; 1875, pl. 21, fig. 4. Dorcadospyris ateuchus (Ehrenberg), Riedel and Sanfilippo, 1970, p. 523 , pl. 15 , fig. 4 .

Eucyrtis bulbosus Renz

Eucyrtis bulbosus Renz, 1974, p. 792, pl. 7, fig 26-29; pl. 12, fig. $15 \mathrm{a}, \mathrm{b}$.

Eucyrtis tenuis (Rust)

Stichocapsa tenuis Rust, 1885, p. 318, pl. 47, fig. 13, 14.

Eucyrtis tenuis (Rust), Foreman, 1975, p. 615, pl. 2I, fig. 7-9.

Lamptonium (?) colymbus Foreman

Lamptonium (?) colymbus Foreman, 1973a, p. 435, pl. 6, fig. 2; pl. 11, fig. 15, 19.

Lamptonium pennatum Foreman

Lamptonium pennatum Foreman, 1973a, p. 436, pl. 6, fig. 3-5; pl. 11 , fig. 13.

Liriospyris parkerae Riedel and Sanfilippo

Liriospyris parkerae Riedel and Sanfilippo, 1971, p. 1590, pl. 2C, fig. 15 ; pl. 5 , fig. 4 .

Liriospyris stauropora (Haeckel)

Trissocyclus stauroporus Haeckel, 1887, p. 987, pl. 83, fig. 5 . Liriospyris stauropora (Haeckel), Goll, 1968, p. 1431, p. 175, fig. $1-3,7$.

Lithochytris vespertilio Ehrenberg

Lithochytris vespertilio Ehrenberg, 1873, p. 239; 1875, pl. 4, fig. 10.

Lithochytris vespertillio Ehrenberg, Riedel and Sanfilippo, 1970, p. 528 , pl. 9 , fig. 8,9 .

Lithocyclia aristotelis (Ehrenberg) group Astromma aristotelis Ehrenberg, 1847, p. 55, fig. 10. Lithocyclia arisotelis (Ehrenberg) group, Riedel and Sanfilippo, 1970 , p. $522 ; 1971$, p. 1588 , pl. 3 A, fig. 4 , 5 .

Lithomitra docilis Foreman Lithomitra docilis Foreman, 1973a, p. 431, pl. 8, fig. 20-22; p. 9, fig. 3-5.

Lithomitra lineata (Ehrenberg) group

Lithocampe lineata Ehrenberg, 1838, p. 130 (partim); 1854, pl. 22, fig. 26; pl. 36, fig. 16 .

Lithomitra lineata (Ehrenberg) group, Riedel and Sanfilippo, 1971 , p. 1600 , pl. 11 , fig. $1-11$; pl. 2I, fig. 14-16; pl. 3E, fig. 14.

Lithopera neotera Sanfilippo and Riedel

Lithopera neotera Sanfilippo and Riedel, 1970, p. 454, pl. 1, fig. 24-26, 28.

Lithopera renzae Sanfilippo and Riedel

Lithopera renzae Sanfilippo and Riedel, 1970, p. 454, pl. 1, fig. 21-23, 27.

Lithopera thornburgi Sanfilippo and Riedel

Lithopera thornburgi Sanfilippo and Riedel, 1970, p. 455, pl. 2, fig. 4-6.

Lychnocanoma elongata (Vinassa)

Tetrahedrina elongata Vinassa, 1900, p. 243, pl. 2, fig. 31 . Lychnocanoma elongata (Vinassa), Sanfilippo and Riedel, in Sanfilippo et al., 1973, p. 221, pl. 5, fig. 19, 20.

Ommatartus antepenultimus Riedel and Sanfilippo

Ommatartus antepenultimus Riedel and Sanfilippo, 1970, p. 521, p. 14 , fig. 4.

Ommatartus antepenultimus Riedel and Sanfilippo, Westberg and Riedel, in press.

Ommatartus hughesi (Campbell and Clark)

Ommatocample hughesi Campbell and Clark, 1944a, p. 23, pl. 3, fig. 12.
Ommatartus hughesi (Campbell and Clark), Riedel and Sanfilippo, 1970, p. 521.

Ommatartus penultimus (Riedel)

Panarium penultimum Riedel, 1957, p. 76, pl. 1, fig. 1.

Ommatarus penultimus (Riedel), Riedel and Sanfilippo, 1970, p. 521.

Ommatartus penultimus (Riedel), Westberg and Riedel, in press.

Phormocyrtis striata exquisita (Kozlova)

Podocyrtis exquisita Koslova, in Kozlova and Gorbovets, 1966, p. 106, p. 17 , fig. 2.

Phormocyrtis striata exquisita (Kozlova), Foreman, 1973a, p. 438, pl. 7 , fig. $1-4,7,8$; pl. 12, fig. 5 .

Phormocyrtis striata striata Brandt

Phormocyrtis striata Brandt, in Wetzel, 1935, p. 55, pl. 9, fig. 12. Phormocyrtis striata Brandt, Riedel and Sanfilippo, 1970, p. 532, pl. 10, fig. 7.

Phormocyrtis striata striata Brandt, Foreman, 1973a, p. 438, pl. 7, fig. $5,6,9$.

Phormostichoartus corona Haeckel

Cyrtophormis (Acanthocyrtis) corona Haeckel, 1887, p. 1462, pl. 77, fig. 15.

Phormostichoartus corona Haeckel, Riedel and Sanfilippo, 1971, p. 1600, pl. II, fig. 13-15; pl. 2J, fig. 1-5.

Pterocodon les Sanfilippo and Riedel

Pterocodon lex Sanfilippo and Riedel, in press.

Siphocampe corbula (Harting)

Lithocampe corbula Harting, 1863, p. 12, pl. 1, fig. 21.

Siphocampe corbula (Harting), Nigrini, 1967, p. 85, pl. 8, fig. 5; pl. 9, fig. 3 .

Spongaster berminghami (Campbell and Clark)

Spongasteriscus berminghami Campbell and Clark, 1944a, p. 30, p. 5 , fig. 1,2 .

Spongaster klingi Riedel and Sanfilippo, 1971, p. 1589, pl. 1D, fig. $8-10$; pl. 4, fig. 7, 8 .

Spongaster berminghami (Campbell and Clark), Sanfilippo and Riedel, 1973, p. 425.

Spongaster berminghami (Campbell and Clark), Riedel and Sanfilippo, in press.

Spongaster pentas Riedel and Sanfilippo

Spongaster pentas Riedel and Sanfilippo, 1970, p. 523, pl. 15, fig. 3 ; in press.

Spongopyle insolita Koxlova group

Spongopyle insolita Kozlova, in Kozlova and Gorbovets, 1966, p. 91 , pl. 4, fig. 11a, b.

Spongopyle insolita Kozlova group, Riedel and Sanfilippo, 1974, p. 780 , pl. 2 , fig. $7-11$; pl. 14 , fig. 4 .

Stichocorys armata (Haeckel)

Cyrtophormis armata Haeckel, 1887, p. 1460, p. 78, fig. 17.

Stichocorys armata (Haeckel), Riedel and Sanfilippo, 1971, p. 1595 , pl. 2E, fig. 13-15.

Stichocorys delmontensis (Campbell and Clark)

Eucyrtidium delmontense Campbell and Clark, 1944a, p. 56, pl. 7, fig. $19,20$.

Stichocorys delmontensis (Campbell and Clark), Sanfilippo and Riedel, 1970, p. 451, pl. 1, fig. 9; Riedel and Sanfilippo, 1971, p. 1595 , pl. $1 \mathrm{~F}$, fig. $5-7$; pl. $2 \mathrm{E}$, fig. 10,11 .

Stichocorys delmontensis (Campbell and Clark), Westberg and Riedel, in press.

Stichocorys peregrina (Riedel)

Eucyrtidium elongatum peregrinuum Riedel, 1953, p. 812, p. 85, fig. 2 .

Stichocorys peregrina (Riedel), Riedel and Sanfillipo, 1970, p. 530. Stichocorys peregrina (Riedel), Westberg and Riedel, in press.

Stichocorys wolffii Haeckel

Stichocorys wolffii Haeckel, 1887, p. 1479, pl. 80, fig. 10.

Stichocorys wolffii Haeckel, Riedel and Sanfillipo, in press.

Stichomitra asymbatos Foreman group

Stichomitra asymbatos Foreman, 1968o, p. 73, pl. 8, fig. 10a-c. Stichomitra asymbatos Foreman group, Riedel and Sanfilippo, 1974 , p. 780 , pl. 15 , fig. 5 ; pl. 10 , fig. $1-7$.

Stylosphaera goruna Sanfilippo and Riedel

Stylosphaera goruna Sanfilippo and Riedel, 1973, p. 521, pl. 1, fig. $10-22$; p. 25 , fig. 9,10 
Stylotorochus alveatus Sanfilippo and Riedel Stylotrochus alveatus Sanfilippo and Riedel, 1973, p. 525, p. 13, fig. 4,5 ; pl. 30 , figs. 3,4 .

Theocampe mongolfieri (Ehrenberg)

Eucyrtidium mongolfiere Ehrenberg, 1854, pl. 36, fig. 18. Theocampe mongolfieri (Ehrenberg), Burma, 1959, p. 329. Theocampe mongolfieri (Ehrenberg), Riedel and Sanfilippo, 1970, p. 536 , p. 12 , fig. 9 .

Theocampe mongolfieri (Ehrenberg), Foreman, 1973a, p. 432, pl. 8, fig. 6; pl. 9, fig. 17.

Theocorys spongoconum Kling

Theocorys spongoconum Kling, 1971, p. 1087, pl. 5, fig. 6 .

Theocyrtis annosa (Riedel)

Phormocyrtis annosa Riedel, 1959, p. 295, pl. 2, fig. 7.

Theocyrtis annosa (Riedel), Riedel and Sanfilippo, 1970, p. 535, pl. 15 , fig. 9 .

\section{REFERENCES}

Brandt, R., 1935. Radiolarien. In Wetzel, O., Die Mikropalaeontologie des Heiligenhafner Kiesletones (Ober-Eözan), Niedersaechsischer geoligischer Verein, Jahresbericht, Hanover, p. 41-81.

Burma, B. H., 1959. On the status of Theocampe Haeckel, and certain similar genera, Micropaleontology, v. 5, p. 325-330.

Campbell, A. S. and Clark, B. L., 1944a, Miocene radiolarian faunas from Southern California, Geological Society of America, Special Paper 51, p. 1-76.

1944b. Radiolaria from Upper Cretaceous of middle California, Geological Society of America, Special Paper 57, p. i-viii + 1-61.

Clark, B. L. and Campbell, A. S., 1942. Eocene radiolarian faunas from the Mt. Diablo area, California, Geological Society of America, Special Paper 39, p. 1-112.

Ehrenberg, C. G., 1838. Uber die Bildung der Kreidefelsen und des Kreidemergels durch unsichtbare Organismen, Königlichen Akademie der Wissenschaften, Abhandlungen, Berlin, p. 59-147.

, 1847. Über die mikroskopischen kieselschaligen Polychystinen als mächtige Gebirgsmasse von Barbados und über das Verhältniss der aus mehr als 300 Neuen Arten bestehenden ganz eigenthümlichen Formengruppe jener Felsmasse zu den jetzt lebenden Thieren und zur Kreidebildung. Eine neue Anregung zur Erforschung des Erdlebens, Königlichen Preussischen Akademie des Wissenschaften, Bericht, Berlin, p. $40-60$.

, 1854. Mikrogeologie: Leipzig (Voss), 374 p. Atlas. 31 p. Fortsetzung (1856), p. 1-88.

, 1873. Grössere Felsproben des Polycystinen-Mergels von Barbados mit weiteren Erläuterungen, Koniglichen Preussischen Akademie der Wissenschaften, Monatsberichte, Berlin, p. 213-263.

, 1875. Fortsetzung der mikrogeologischen Studien als Gesammt-Uebersicht der mikroskopischen Paläontologie gleichartig analysirter Gebirgsarten der Erde, mit specieller Rücksicht auf den Polychstinen-Mergel von Barbados, Königlichen Akademie der Wissenschaften, Abhandlungen, Berlin, p. 1-226.

Foreman, H. P., 1968. Upper Maestrichtian Radiolaria of California, The Palaeontological Association, London, Special Paper 3, p. i-iv + 1-182.

, 1971. Cretaceous Radiolaria. In Winterer, E. L. Riedel, W. R., et al., Initial Reports of the Deep Sea Drilling Project, v. 7, Part 2: Washington (U.S. Government Printing Office), p. 1673-1693.

1973a. Radiolaria of Leg 10 with systematics and ranges for the families Amphipyndacidae, Artostrobiidae, and Theoperidae. In Worzel, J. L., Bryant, W., et al., Ini- tial Reports of the Deep Sea Drilling Project, v. 10: Washington (U.S. Government Printing Office), p. 407474.

1973b. Radiolaria from DSDP Leg 20. In Heezen, B. C., MacGregor, I. D., et al., Initial Reports of the Deep Sea Drilling Project, v. 20: Washington (U.S. Government Printing Office), p. 249-305.

1975. Radiolaria from the North Pacific, Deep Sea Drilling Project, Leg 32. In Larson, R. L., Moberly, R., et al., Initial Reports of the Deep Sea Drilling Project, v. 32: Washington (U.S. Government Printing Office), p. 579676.

Goll, R. M., 1968. Classification and Phylogeny of Cenozoic Trissocyclidae (Radiolaria) in the Pacific and Caribbean basins, part I, Journal of Paleontology, v. 42, p. 14091432.

Haeckel, E., 1887. Report on the Radiolaria collected by $\mathrm{H}$. M.S. Challenger during the years 1873-76, Report on the Scientific Results of the Voyage of H.M.S. Challenger, Zoology, v. 18, p. 1-1303.

Harting, P., 1863. Bijdrage tot de kennis der mikroskopische faune en flora van de Banda-Zee, Koninklijke Akademie van Wetenschappen, Verhandelingen, 10, Amsterdam, p. 1-34.

Kling, S. A., 1971. Radiolaria. In Fischer, A. G., Heezen, B. C. et al., Initial Reports of the Deep Sea Drilling Project, v. 6: Washington (U. S. Government Printing Office), p. 1060-1117.

Kozlova, G. E. and Gorbovets, A. N., 1966. Radiolyarii verkhnemelovykh i verkhneeotsenovykh otlozhenii ZapadnoSibirskoi Nizmennosti. Trudy Vsessoyuznogo Neftyanogo Nauchno-Issledovateljskogo Geologorazvedochnogo Instituta (VNIGRI, no. 248, p. 1-159).

Moore, T. C., Jr., 1971, Radiolaria. In Tracey, J. L., Jr., Sutton, G. H., et al., Initial Reports of the Deep Sea Drilling Project, v. 8: Washington (U. S. Government Printing Office), p. 727-775.

1972. Mid-Tertiary evolution of the radiolarian genus Calocycletta, Micropaleontology, v. 18, p. 144-152.

Nigrini, C., 1967. Radiolaria in pelagic sediments from the Indian and Atlantic Ocean, Bulletin of the Scripps Institution of Oceanography, University of California, San Diego, La Jolla, California, v. 11, p. 1-125.

Petrushevskaya, M. G. and Kozlova, G. E., 1972. Radiolaria. In Hayes, D. E., Pimm, A. C., et al., Initial Reports of the Deep Sea Drilling Project, v. 14: Washington (U. S. Government Printing Office), p. 495-648.

Renz, G. W., 1974. Radiolaria from Leg 27 of the Deep Sea Drilling Project. In Veevers, J. J., Heirtzler, J. R., et al., Initial Reports of the Deep Sea Drilling Project, v. 27: Washington (U. S. Government Printing Office), p. 769841.

Riedel, W. R., 1953. Mesozoic and late Tertiary Radiolaria of Rotti, Journal of Paleontology, v. 27, p. 805-813.

1957. Radiolaria: a preliminary stratigraphy, $R e-$ ports of the Swedish Deep-Sea Expedition, v. 6, p. 5996.

1959. Oligocene and Lower Miocene Radiolaria in tropical Pacific sediments, Micropaleontology, v. 5, p. 285-302.

Riedel, W. R. and Sanfilippo, A., 1970. Radiolaria, Leg 4, Deep Sea Drilling Project. In Bader, R. G., Gerard, R. D. et al., Initial Reports of the Deep Sea Drilling Project, v. 4: Washington (U. S. Government Printing Office), p. 503575 .

1971. Cenozoic Radiolaria from the western tropical Pacific, Leg 7. In Winterer, E. L., Riedel, W. R., et al., 
Initial Reports of the Deep Sea Drilling Project, v. 7: Washington (U. S. Government Printing Office), p. 15291672.

1974. Radiolaria from the Southern Indian Ocean, DSDP Leg 26. In Davis, T. A., Luyendyk, B. P., et al., Initial Reports of the Deep Sea Drilling Project, v. 26: Washington (U. S. Government Printing Office), p.771-814. in press. Stratigraphy and evolution of tropical Cenozoic radiolarians, Micropaleontology.

Rüst, D., 1885. Beiträge zur Kenntniss der fossilen Radiiolarien aus Gesteinen des Jura, Paleontographica, v. 31, ser. 3, p. 273-321.

Sanfilippo, A. and Riedel, W. R., 1970. Post-Eocene "closed" theoperid radiolarian, Micropaleontology, v. 16, p. 446-462. , 1973. Cenozoic Radiolaria (exclusive of theoperids, artostrobiids and amphipyndacids) from the Gulf of Mexico, DSDP Leg 10. In Worzel, J. L., Bryant, W., et al., Initial Reports of the Deep Sea Drilling Project, v. 10: Washington (U. S. Government Printing Office), p. 475611 .
, in press. Radiolaria from the northeastern Atlantic Ocean, DSDP Leg 48. In Montadert, L., Roberts, D. G. et al., Initial Reports of the Deep Sea Drilling Project, v. 48: Washington (U. S. Government Printing Office), p. 493512.

Sanfilippo, A., Burckle, L. H., Martini, E. and Riedel W. R., 1973. Radiolarians, diatoms, silicoflagellates and calcareous nannofossils in the Mediterranean Neogene, Micropaleontology, v. 19, p. 209-234.

Squinabol, S., 1903. Le Radiolarie di noduli selciosi nella Scaglia degli Euganei, Revista Italiana di Paleontologia, v. 9, p. 105-150.

1904. Radiolarie cretacee degli Euganei, Atti $e$ Memorie della $R$. Accademia di Scienze Lettere ed. Arti in Padova, v. 20, p. 171-244.

Vinassa De Regny, P. E., 1900. Radiolaria miocenici italiani, Memorie della $R$. Accademia delle Scienze dell'Istituto di Bologna, v. 8, p. 565-595.

Westberg, M. J. and Riedel, W. R., in press. Accuracy of radiolarian correlations in the Pacific Miocene, Micropaleontology, v. 23. 



\section{PLATE 1}

In the figure explanations, the sample numbers and slide designations in the form "Sl.1" indicate preparations in our collection, and designations in the form " $\mathrm{K} 26 / 3$ " indicate England Finder positions of the illustrated specimens on the slides. (Unless otherwise indicated, magnifications are $\times 280$.)

Figure 1 Stylosphaera goruna Sanfilippo and Riedel. Sample 415A-6-2, 118-120 cm, Sl.2, K26/3.

Figure 2 Saturnalin, gen. et sp. indet.

Sample 415A-6-2, 118-120 cm, Sl.1, T19/4, ×190.

Figure 3 Spongopyle insolita Kozlova group.

Sample 415A-6-2, 118-120 cm, Sl.1, D12/1.

Figure 4 Spyrid, gen. et sp. indet.

Sample 415A-6-2, 118-120 cm, Sl.1, B14/0.

Figure 5 Amphiternis sp. cf.? Stichomitra alamedaensis (Campbell and Clark).

Sample 415A-6-2, 118-120 cm, Sl.2, H23/0.

Figure 6 Bekoma campechensis Foreman.

Sample 415A-5-1, 43-45 cm, Sl.2, E64/4, ×190.

Figure $7 \quad$ Bekoma divaricata Foreman.

Sample 415A-5, CC, Sl.1, ×46/0, ×190.

Figure $8 \quad$ Buryella pentadica Foreman.

Sample 415A-6-2, 118-120 cm, Sl.1, P41/0.

Figure 9 Buryella tetradica Foreman.

Sample 415A-6-2, 118-120 cm, Sl.1, N31/3.

Figure 10 Lamptonium pennatum Foreman.

Sample 415A-6-2, 118-120 cm, Sl.1, T28/1.

Figures 11, 12 Lychnocanoma spp.

11. Sample 415A-5, CC, S1.2, 037/0.

12. Sample 415A-6-2, $118-120 \mathrm{~cm}, \mathrm{Sl} .1, \mathrm{~V} 18 / 1$.

Figure 13 Phormocyrtis striata exquisita (Kozlova). Sample 415A-5, CC, Sl.2, B10/1.

Figure 14 Pterocodon sp. aff. P. lex Sanfilippo and Riedel. Sample 415A-6-2, 118-120 cm, Sl.2, V29/0. 
PLATE 1
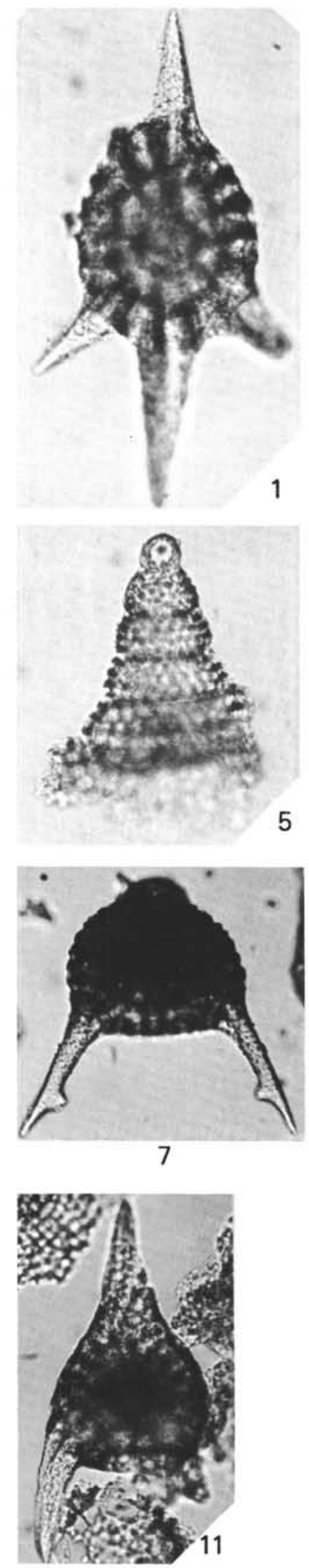
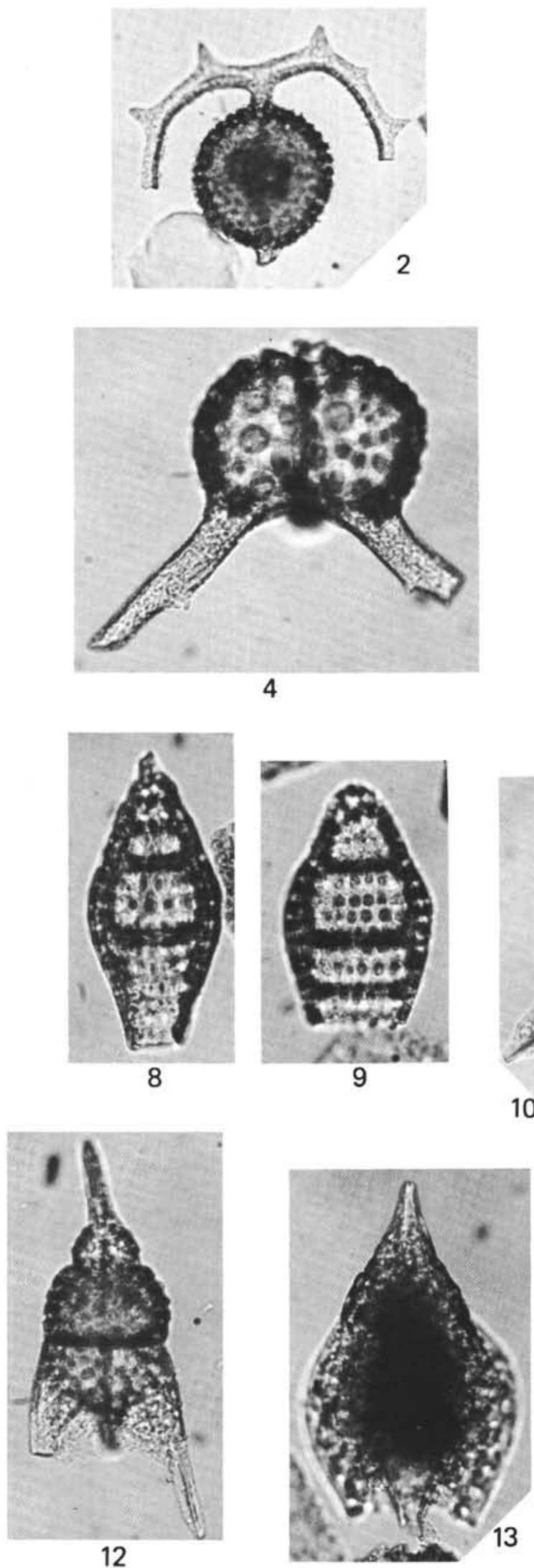
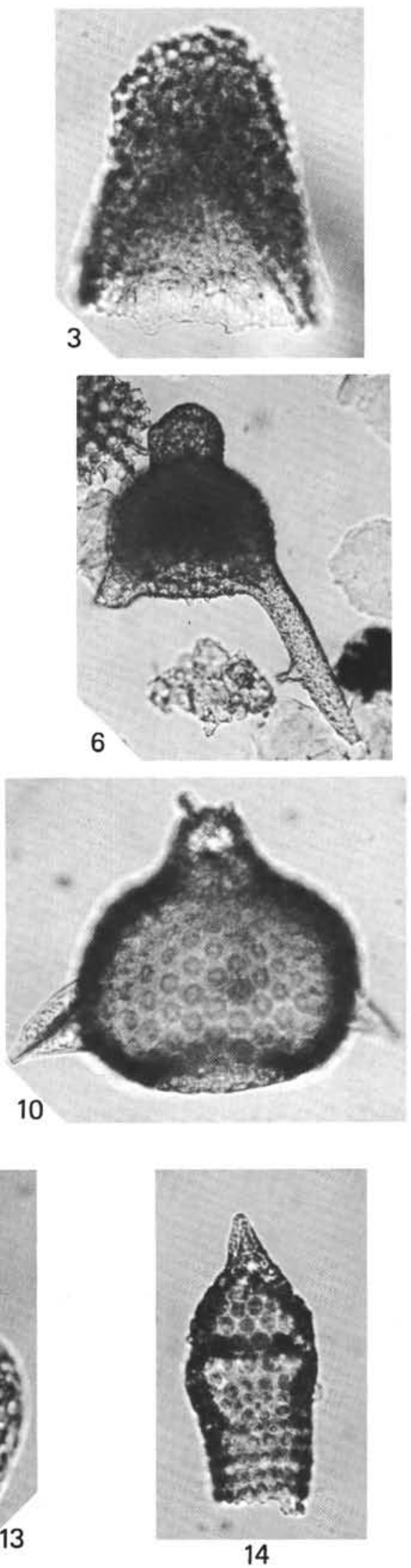